\title{
Type-safe Dynamic Protocol Composition in Jgroup/ARM
}

\author{
Hein Meling \\ Department of Electrical Engineering and \\ Computer Science \\ University of Stavanger, 4036 Stavanger, Norway \\ hein.meling@uis.no
}

\author{
Alberto Montresor \\ Dip. di Ingegneria e Scienza dell'Informazione \\ University of Trento, via Sommarive 14, 38050 \\ Trento, Italy \\ alberto.montresor@unitn.it
}

\begin{abstract}
Protocol composition is a common approach to structure protocols used by networked applications, and typically a vertically layered approach is taken. This paper presents an alternative approach, where the protocol composition is a weakly-coupled set of protocol modules organized in a nonhierarchical structure. Protocol modules are dynamically constructed at runtime. The approach is designed for systems that involves multiple communicating entities and multicast style interactions are supported, making the approach suitable for building reliable network applications. The main advantage of the approach is type-safety and that modules in the same composition communicate by direct interaction, whereas other frameworks typically use a vertically layered protocol stack, forcing all messages/events to pass through all intermediate layers introducing unnecessary delays.
\end{abstract}

\section{Categories and Subject Descriptors}

C.2.8 [Computer Systems Organization]: Computer Communication Networks-Distributed Systems; D.1.3 [Software]: Concurrent Programming - Distributed Programming; D.2.12 [Software]: Software Engineering-Distributed Objects

\section{Keywords}

Dynamic Protocol Composition, Group Communication, Replication, Recovery

\section{INTRODUCTION}

Networked computer systems are prevalent in most aspects of modern society, and we have become dependent on them to perform many critical tasks. Making such systems dependable is an important goal. Yet, dependability issues are often neglected due to the complexities of the techniques involved. Modularization is a well-known principle for simplifying complex systems, and combined with middleware to support replication, it is possible to construct fault-tolerant applications with significantly smaller costs.

Permission to make digital or hard copies of all or part of this work for personal or classroom use is granted without fee provided that copies are not made or distributed for profit or commercial advantage and that copies bear this notice and the full citation on the first page. To copy otherwise, to republish, to post on servers or to redistribute to lists, requires prior specific permission and/or a fee.

MAI'09 Lisbon, Portugal

Copyright 2009 ACM 978-1-60558-489-8/09/06 ...\$10.00.
This paper presents the protocol composition framework of Jgroup/ARM [9], a middleware platform for developing and operating dependable distributed applications. Jgroup integrates the distributed object model of Java remote method invocations (RMI) with the object group communication paradigm, enabling the construction of groups of replicated server objects, denoted replicas, that provide dependable services to clients. The Autonomous Replication Management (ARM) framework provides mechanisms for deploying replicas to host processors and recovering from failures.

Jgroup/ARM is aimed at simplifying the development of dependable network information services. One part of this simplification is accomplished through modularization of protocol modules and composing them into a complete protocol stack. Protocols in Jgroup/ARM usually involves multiple communicating entities, i.e. all members of a replicated object group, and specialized multicast interactions are supported for interactions with peers in the same group. Protocol modules in the same composition (i.e., same process) communicate by direct interaction. Using this framework, a dependable service can easily construct and configure its own protocol composition dynamically at deployment time. Each protocol module in the composition can be parameterized according to the service dependability requirements. Adding new protocols to the system is also very easy.

Paper structure: Section 1.1 discusses previous works on protocol architectures and relates these to the approach taken by Jgroup/ARM. Section 2 briefly presents Jgroup/ARM. Section 3 introduces the concepts on which the protocol composition framework is based, and illustrates a sample protocol stack. Section 4 details the ways in which protocol modules can communicate, both internally and externally. Finally, in Section 5 the dynamic composition of protocol modules is discussed and Section 6 concludes the paper.

\subsection{Introduction to Protocol Architectures}

Protocol composition is traditionally based on layered protocol stacks. However, in the last decade, micro-protocols have become increasingly popular, as they enable a more flexible approach to protocol composition. To accomplish this, micro-protocol frameworks restrict their protocol layers to follow a specific model, rather than building protocols in an ad hoc manner. These restrictions include: (i) the protocol layers have to communicate using events that travel up or down the protocol stack, and (ii) the layers cannot share any state. This way protocols become more maintainable and configurable as new protocols can easily be added to the system. The cost however, is reduced performance.

Micro-protocols were first introduced in the $x$-kernel [7], 
and have since been used in a variety of systems, including group communication systems such as Ensemble [5], Horus [15], JavaGroups [2], Cactus [6] and Appia [10]. Ensemble, Horus, JavaGroups and Appia follow a strictly vertical stack composition, where events must pass through all layers in the stack. In the Horus system, a protocol accelerator [14] implements optimizations that reduce the effects of protocol layering. The limitation of these optimization techniques is that the set of protocols to be bypassed must be well-defined, and the optimizations were hand-coded into the protocol stack. Thus, it reduces the configurability of the microprotocol framework. Similar optimizations are also feasible with Ensemble. Both Appia and JavaGroups are also based on micro-protocols in its purest form, since none of the optimizations implemented in Horus and Ensemble are available. That means that every event has to pass through all intermediate layers, even when the event is not being processed by all of them. The Cactus micro-protocol framework is conceptually similar to the protocol composition framework discussed in this paper. Each layer has to register its interest in the events of other layers, and protocols can be constructed according to formal rules, such as a dependency graph. Thus, such a protocol stack does not follow a strict vertical composition. An advantage of the Jgroup protocol framework over JavaGroups, Appia and the Cactus system is type-safety. Events are passed by means of method calls on a set of well-defined interfaces for the various modules (layers), whereas other systems have to implement a common handler method in each layer which takes care of demultiplexing the received events based on the type of the events. In Jgroup, events are passed directly to the appropriate event handler. Another advantage of Jgroup over the Cactus system is the possibility to specify interception rules, enabling a module to delay and/or modify events from another module. The SAMOA [12] framework is also conceptually similar to the approach in this paper. The main differences are that SAMOA supports concurrency and asynchronous stack internal interactions, whereas Jgroup uses synchronous interactions and leaves concurrency a non-framework issue. Synchronous interaction is a simpler approach, and makes it easier for developers to write protocol modules. Both the Neko [13] protocol prototyping framework and Jgroup/ARM uses an approach based on dependency injection [4]. Kompics [3] use generics to ensure strongly typed channels for event delivery, but does not leverage dependency injection for connecting components, and thus protocol developers need to manually connect components.

\section{THE JGROUP/ARM MIDDLEWARE}

Jgroup [9] integrates the Java RMI distributed object models with the group communication paradigm and autonomous fault treatment. Jgroup provides three core services aimed at simplifying coordination among replicas: a partition-aware group membership service (PGMS), a group method invocation service (GMIS) and a state merging service (SMS).

The task of the PGMS is to provide replicas with a consistent view of the group's current membership, to enable replica coordination. Reliable communication between clients and the object group take the form of group method invocations (GMI), that result in methods being executed by the replicas in the group. To clients, GMI interactions are indistinguishable from standard Java RMI: clients interact with the group through a group proxy that acts as a representa-

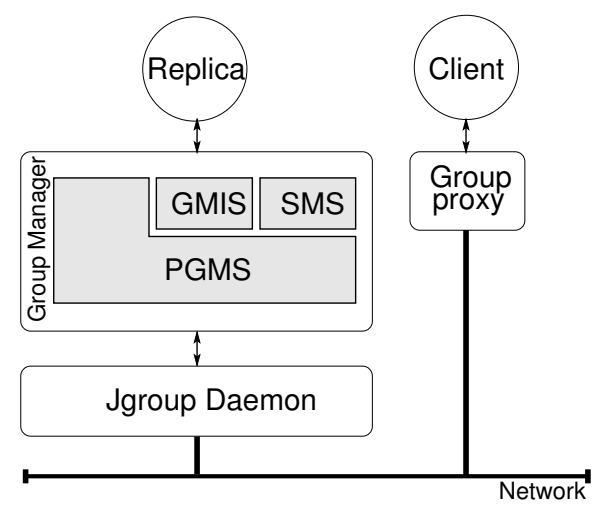

Figure 1: Overview of Jgroup services.

tive object for the group, hiding its composition. The group proxy maintains information about the replicas composing the group, and handle invocations on behalf of clients by establishing communication with one or more replicas and returning the result to the invoker. This form of GMI is called External GMI (EGMI). On the server side, the GMIS enforce reliable communication among replicas within the group and are called Internal GMI. Finally, the task of SMS is to support developers in re-establishing a global shared state after merging from a network partition.

Fig. 1 gives a high-level overview of the composition of the core Jgroup services. The main component of Jgroup is the Jgroup daemon; it implements basic group communication services such as failure detection, group membership and reliable communication. Replicas must connect to a Jgroup daemon to access to the group communication services. Each replica is associated with a group manager (GM), whose task is to act as an interface between the Jgroup daemon and the replica.

The ARM framework [8] provides mechanisms for automated fault treatment and management activities such as distributing replicas on sites and nodes, and recovering from failures, reducing the need for human intervention. These mechanisms are essential to operate a system with strict dependability requirements, and are largely missing from existing group communication systems $[11,10,2]$. Much of the ARM functionality is implemented by separate protocol modules integrated into the GM component.

\section{PROTOCOL MODULES}

The group manager (GM) is the glue between an application and the core group communication services; it encapsulates all protocol modules associated with the application. It allows the application to interface with Jgroup services to perform group-specific tasks. The GM is based on an eventdriven non-hierarchical composition model, and consists of a set of weakly coupled protocol modules. Each protocol module implements a group-specific function, which may require the collaboration of all group members, e.g. the membership service. In fact, all the basic Jgroup services discussed in Section 2 and several other generic group-specific functions are implemented as GM protocol modules.

The advantages of weakly coupled protocol modules over a strictly vertically layered architecture, is that events being passed from one layer (module) do not have to be processed by any intermediate layers. Events can simply be passed from one module to another without any processing delay 
and addition/removal of header fields, thus also reducing the complexity of implementing a module. Our approach is also flexible in that a module can intercept command$\mathrm{s} /$ events from another module, delay and/or modify them, before delivery to the destination module. Interception rules are specified inline in the modules using annotations, and the corresponding implementations must adhere to these rules.

Protocol modules communicate with the application, or other modules, by means of commands (downcalls) and events (upcalls) through a set of well-defined interfaces. Typically, a module provides a set of services to other modules and/or the application, and requires another set of services from other modules to perform its services. A module may also substitute the services provided by another module, by intercepting, delaying and/or modifying the commands/events passed on to the substituted module.

Each module implements one or more well-defined service interfaces, through which the module can be controlled, and it may also generate events to listening modules (or the application) through one or more listener interfaces. Usually, a module implements one service interface and provide events to other modules through one listener interface. As an example, consider the MembershipModule which is defined by the MembershipService and MembershipListener interfaces, shown in Fig. 2. Replicas can access the service interfaces of protocol modules by querying the GM. However, to be notified of events generated by a module, a replica only needs to implement the module's listener interface.

The set of protocol modules required by an application is configured through the ARM policy management [8]. Based on this configuration, the protocol modules are constructed dynamically at runtime. The advantage of dynamic construction is that it enables developers to easily build generic group-specific functions and augment the system with new modules without having to recompile the complete framework. There is no strict ordering in which the modules have to be constructed, except that the set of required modules must have been constructed a priori. During construction, each module is checked for structural correctness, and required modules are constructed on-demand.

Fig. 2 illustrates a protocol composition containing the basic Jgroup services, except the GMIS. For readability only the most important commands/events are shown in the interfaces. The DispatcherModule is responsible for queuing and dispatching events to/from the daemon, and is the interface between the protocol modules and the daemon. The MulticastModule implements the MulticastService through which other modules (and the replica) can send multicast messages to the group members. To receive messages, a module must implement the MulticastListener interface. The main task of the MulticastModule is to multiplex and demultiplex messages to/from the internal modules or the replica. The actual lowlevel multicast is performed by the daemon. Other modules (or the replica) can join() or leave() a group by invoking the MembershipService interface, which is implemented by the MembershipModule. Variations in the group membership are reported through viewChange() events. Any number of modules, and the replica, may register its interest in such events simply by implementing the MembershipListener interface. The MembershipModule mainly keeps track of various state information and provides an interface to the PGMS, whereas the view agreement protocol [11] is implemented in the daemon. The DispatcherModule, MulticastModule and Mem-

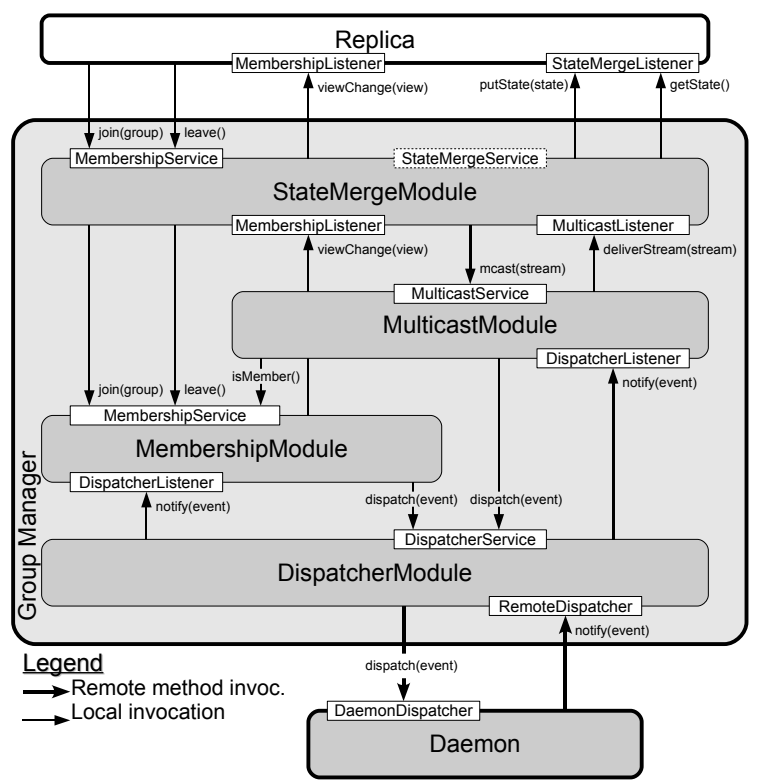

Figure 2: A sample group manager composition with the basic Jgroup services.

bershipModule are mandatory, and must always be included to support group communication.

Note that the StateMergeModule implements the MembershipService interface, and provides events through the MembershipListener interface. This is since the StateMergeModule substitutes the membership service by intercepting and delaying the delivery of viewChange() events to the replica until after the state has been merged. The main task of the StateMergeModule is to drive the state reconciliation protocol by calling getState() and putState() on the StateMergeListener interface to obtain and merge the state of the replicas. It also handles leader election and information diffusion. State reconciliation is only activated when needed, i.e. in response to viewChange() events generated by the MembershipModule. Hence, the StateMergeService interface (dashed box) does not provide commands as a means for activating it. The StateMergeModule also requires both the MembershipModule and the MulticastModule, and substitutes the MembershipModule.

\section{MODULE INTERACTIONS}

Protocol modules may interact in a number of different ways, both with external entities and other protocol modules. Hence, to construct the protocol modules dynamically, it is necessary to understand how the modules can interact so as to dynamically establish the necessary links between them.

Fig. 3 illustrates inter-module and replica-to-module interactions. Inter-module interactions may occur both within the same GM, and also across distinct GMs. Mostly, only GMs that belong to the same group needs to communicate. GMs belonging to the same group should be composed of an identical set of protocol modules. The arrows in Fig. 3 represents a may communicate relation. That is, a module may or may not communicate with another module in one or both directions. The replicas may also interact directly with one or more of the modules within its local GM, without passing through any intermediate modules. The thicker arrows represent remote interaction between peer modules.

Four distinct interaction styles involving protocol modules 


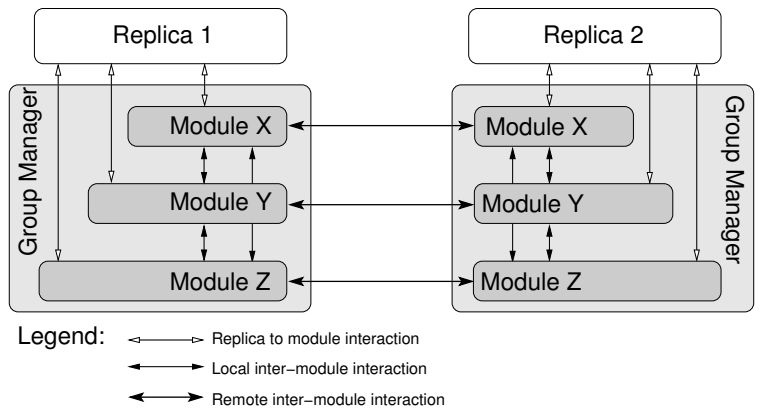

Figure 3: Inter-module and replica-to-module interactions.

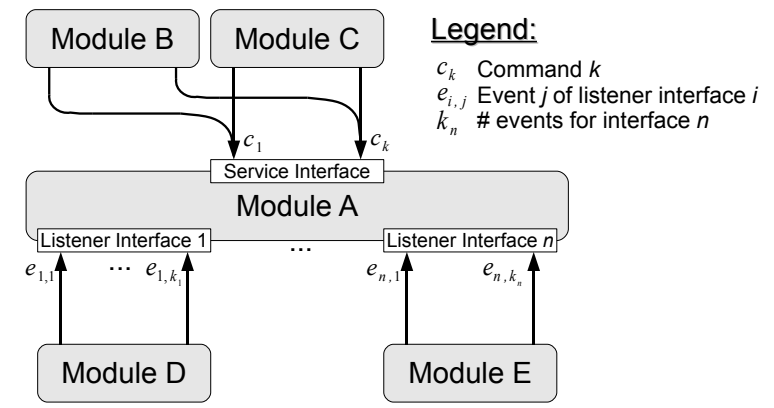

Figure 4: A generic view of the interfaces used for local inter-module interactions.

are listed below. The first three are shown in Fig. 3 .

1. Local inter-module interactions between modules of the same GM.

2. Remote inter-module interactions between peer modules in distinct GMs.

3. Interactions between the replica and its local modules.

4. Interactions between an external entity and a module.

The last interaction style allows a module to notify or to be notified by an external entity. Below, each of these interaction styles are discussed individually. Although commonplace, application-level replica-to-replica interactions are not considered here.

Local Inter-module Interactions As mentioned above, the GM is composed of a collection of protocol modules, each of which may provide a service to other modules in the same GM. In addition, a protocol module may also listen to events from other modules. Fig. 4 illustrates a generic view of the internal inter-module interaction interfaces, through which local protocol modules communicate. In the figure, the service interface implemented by module $\mathrm{A}$ is used by modules $\mathrm{B}$ and $\mathrm{C}$ within the same GM to invoke commands offered through the service interface (e.g. to join() a group). Module A also implements a set of listener interfaces through which it can be notified of events generated by modules D and $\mathrm{E}$ (e.g. a viewChange() event.)

A module must implement at least one service interface, but may also implement more than one service (not shown in Fig. 4). Implementing multiple service interfaces is useful when a module intercept and substitute the services of another module, e.g. the StateMergeModule in Fig. 2. For most other circumstances a module should implement only a single service interface to encourage reuse.
The service interface typically contains one or more commands $\left(c_{1}, \ldots, c_{k}\right)$, that can be invoked by the replica or other modules. The service interface may also be empty in that it does not provide any commands (methods). Such empty interfaces are often called marker interfaces, and serve to identify the module internally in the GM. The dashed box around the StateMergeService interface in Fig. 2 is one example of an empty marker interface.

A module may have one or more associated listener interfaces through which module generated events can be passed to its listeners (other modules or the replica). Fig. 2 illustrates the use of multiple listener interfaces; the StateMergeModule generates events through both StateMergeListener and MembershipListener, since the StateMergeModule substitutes the MembershipModule. Usually however, a module generates events through a single listener interface (see Fig. 4). A module without any associated listener interfaces is useful only when the module provide service commands. A module may receive events $\left(e_{j, 1}, \ldots, e_{j, k_{j}}\right)$ generated by other modules by implementing the listener interface $j$.

The service and listener interfaces are defined in terms of Java interfaces (ensuring type safety), and arrows in Fig. 4 represents Java methods (commands/events).

Remote Inter-module Interactions Modules in one GM may interact with its remote peer modules in other GMs of the same group. Two approaches can be used by module developers for interaction between peer modules:

- Message multicasting (MulticastModule)

- Internal group method invocations (InternalGMIModule). The advantage of the former approach is primarily efficiency, since it adds no overhead to the messages being sent by the module, except for a small header used to route multicast messages to the appropriate peer modules. The drawback with message multicasting is that module complexity increases, since the developer must implement marshalling and unmarshalling routines for the different message types to be exchanged between peer modules.

Contrarily, the InternalGMIModule takes care of marshalling and unmarshalling, reducing the module complexity to pure algorithmic considerations. The InternalGMIModule does however impose an additional overhead compared to that of message multicasting. The overhead is mostly due to the use of dynamically generated proxies $[1$, Ch.16]. Albeit not confirmed through measurements, the expected overhead imposed by the proxy mechanism is small compared to the communication latencies between the peer modules. Details of the workings of the InternalGMIModule as a means for communication between peer modules is given in [8].

Replica to Module Interactions The replica implementation may interact with the local modules. Fig. 5 shows a generic view of the replica-to-module interactions. A replica may choose to listen to an arbitrary set of events generated by its associated protocol modules. To accomplish this, the replica must implement the listener interfaces associated with the modules whose events are of interest. The replica may also choose to not implement any listener interfaces if it does not need to process events generated by modules. In a similar manner, the replica may invoke any one of the commands provided through the service interfaces of the protocol modules associated with the replica.

As Fig. 5 shows, various combinations of using services and listening to events are possible. The replica may both listen to events of a module, and invoke its service commands 


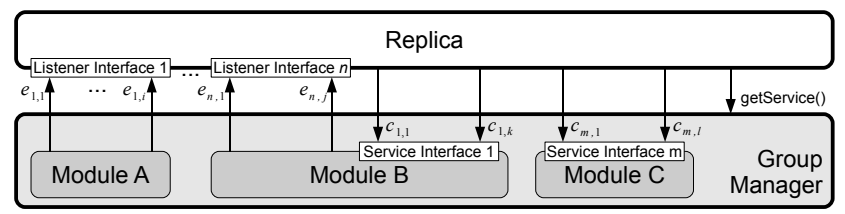

Legend:

$c_{m, l}$ Command $/$ of service interface $m$

$e_{n, j}^{m, l}$ Event $j$ of listener interface $n$

Figure 5: A generic view of the replica-to-module interaction interfaces.

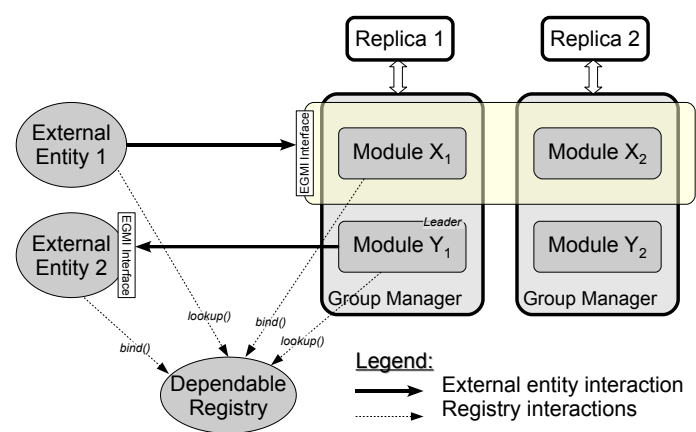

Figure 6: External entity to module interactions.

(middle), or it may just listen to its events (left), or just invoke its service commands (right).

Establishing the connections between the replica and its associated set of protocol modules is done through the GroupManager object. The GroupManager wraps the protocol modules and acts as an interface between the modules and the replica. Initially, when the replica requests group communication support it will invoke the getGroupManager() factory method, passing its own reference (this). Given this reference, the GM establishes upcall connections between the replica and the modules whose listener interfaces are implemented by the replica. On the other hand, establishing connections between the replica and the service interfaces of modules are done on-demand by the replica itself. This is accomplished using the GroupManager.getService() method shown in Fig. 5. Given a reference to the service interface of a module, the replica can easily invoke its commands.

External Entity to Module Interactions Protocol modules may also interact directly with (possibly replicated) external entities. For instance, a protocol module could invoke methods on an external entity or vice versa. This interaction style is useful for a number of purposes, such as event logging, event notifications or triggering some action, e.g. recovery or upgrade [8].

External entities and modules can interact in both directions, as shown in Fig. 6. External Entity 1 invokes Module $\mathrm{X}$ to perform some operation implemented by the module, whereas External Entity 2 allows a module to invoke methods on it to perform some operation. Interaction with external entities relies on the dependable registry for looking up the reference of the external entity (or the module) with which to communicate. Prior to such lookups, the receiving end must bind() its reference in the dependable registry. The two interactions shown in Fig. 6 are both based on EGMI, and hence the receiving end must include the ExternalGMIModule in its set of protocol modules.

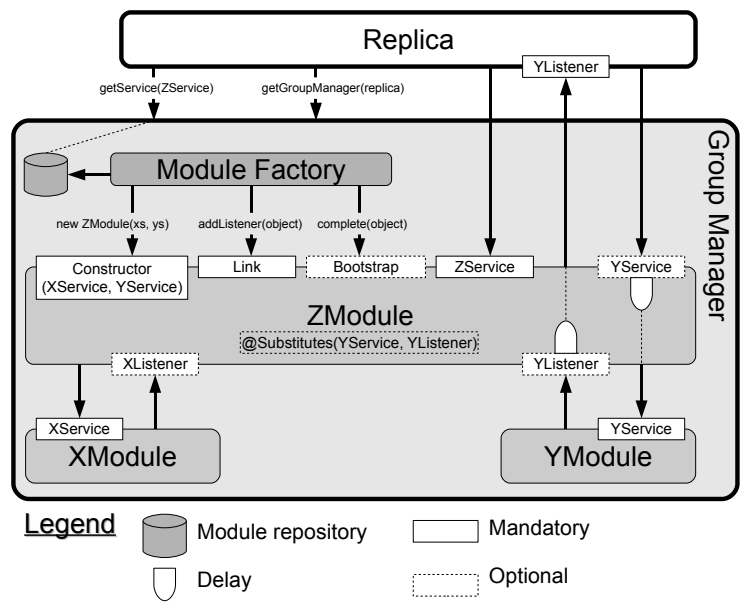

Figure 7: The module factory and interfaces used for module construction.

\section{DYNAMIC MODULE CONSTRUCTION}

The group manager encapsulates the set of protocol modules associated with an application. Protocol modules are configured using an application-specific replication policy [8]. The policy supports specifying the set of protocol modules to be constructed, as well as supplying configuration parameters to the modules, e.g. timeout values and redundancy level.

Protocol modules are constructed dynamically at runtime based on the policy of the application requesting a protocol composition. This is essentially all that an application developer needs to know about the construction of protocol modules. However, a module developer needs to have more intimate knowledge of the architecture which simplifies the following tasks:

- Automatic construction of protocol modules.

- Establishing links between dependent modules.

- Linking the replica with its dependent modules.

- Reconfiguration of links for module substitution.

The dynamic construction facility requires that module implementations adhere to these rules:

1. Must contain a single constructor, whose signature is the set of services required by the module.

2. Must implement the Link interface.

3. May implement the Bootstrap interface.

4. May implement listener interfaces of other modules.

5. May declare that it substitutes the services/listeners provided by another module.

Fig. 7 illustrates the rules in terms of interfaces. Solid boxes indicate required interfaces, while dashed boxes denote optional interfaces which may be implemented by a module depending on its requirements.

Module Instantiation As shown in Fig. 7, ZModule requires two other services, XService and YService, which are implemented by XModule and YModule, respectively. These two modules must have been constructed prior to ZModule, and are thus passed to the ZModule constructor. The module factory use reflection [1, Ch.16] to examine the constructor signature of the ZModule to find its required dependencies, 
and queries the module repository to obtain those module instances. If a required module is not found, it will be created on-demand and stored in the repository.

Construction follows the bottom-up order specified in the replication policy. In Fig. 2, this means that the DispatcherModule is constructed first, followed by the MembershipModule and so on. Note that the DispatcherModule does not depend on other modules, but connects with the daemon.

Link Configuration Once all the protocol modules associated with an application have been instantiated, links between the modules are established by the module factory through the mandatory Link interface. The addListener() method shown in Fig. 7 serves two primary purposes:

- Establish upcall links with other modules; links are only established with modules implementing the listener interface(s) associated with the module.

- Perform bootstrap operations that cannot be performed during module construction.

In Fig. 7 the object passed to the addListener() method may be either the replica object or a module. Note that the replica object is always passed to the addListener() method, independent of it implementing the listener interface associated with the module. Thus the module can exploit the replica reference type as a means to obtain necessary configuration data from the replication policy, e.g. timeout values, to configure/bootstrap the module. If the replica object does not implement the listener interface of the module, it cannot receive any events from the module. Furthermore, the addListener() method may be invoked several times for distinct modules, allowing multiple modules to receive the same set of events. The order in which addListener() is invoked follows the construction order defined above, with the replica object passed in last.

Bootstrapping Some modules may need to perform additional bootstrap operations after all the links have been established. The final task of the module factory is to find modules that implement the optional Bootstrap interface, and invoke its complete() method to finalize bootstrapping. For instance, the application could configure its replication policy to automatically join() its group during the bootstrap phase, simplifying the replica implementation even further. Joining the group requires that all the links have been set up between all the protocol modules, and hence it cannot be bootstrapped through the Link interface.

Event Interception As advocated initially in this paper, some modules need to intercept commands/events originated in other modules. Such interception may be necessary for a number of reasons, e.g. if delivery of events must be delayed until after the intercepting module has completed its tasks. For example, a total ordering module needs to delay the delivery of messages pending agreement among group members on the sequence in which to deliver messages.

Modules that wish to intercept the commands/events of another module must declare that it substitutes the other module. The eSubstitute declaration uses annotations $[1$, Ch.15] to indicate which service and listener interfaces to substitute. As shown in Fig. 7, the ZModule substitutes both interfaces associated with the YModule. The module factory will analyze the substitute declarations and reconfigure the links accordingly, hiding the presence of the YModule from other modules and the replica. Implementing a module which substitutes another can be accomplished by inheriting from the substituted module, or by wrapping it.

\section{CONCLUSIONS AND FUTURE WORK}

In this paper, the design and implementation of a protocol composition framework for Jgroup/ARM has been presented. The main feature is the direct communication between protocol modules, saving costly processing in intermediate modules. It also supports dynamic construction of protocol compositions based on a simple configuration file. The protocol composition problem seems to lend itself nicely to new ideas from aspect-oriented programming and annotations, and we aim to pursue these topics in future work.

\section{REFERENCES}

[1] K. Arnold, J. Gosling, and D. Holmes. The Java Programming Language. Addison-Wesley, 2005. 4th ed.

[2] B. Ban. JavaGroups - Group Communication Patterns in Java. Technical report, Dept. of Computer Science, Cornell University, July 1998.

[3] J. Dowling and C. Arad. Kompics Programming Manual, Apr. 2009. Version 0.4.1.

[4] M. Fowler. Inversion of Control Containers and the Dependency Injection pattern, Jan. 2004. http://www.martinfowler.com/articles/injection.html.

[5] M. Hayden. The Ensemble System. PhD thesis, Dept. of Computer Science, Cornell University, Jan. 1998.

[6] M. A. Hiltunen and R. D. Schlichting. The Cactus Approach to Building Configurable Middleware Services. In Proc. Workshop on Dep. Sys. Middleware and Group Comm., Nuremberg, Germany, Oct. 2000.

[7] N. C. Hutchinson and L. L. Peterson. The $x$-Kernel: An architecture for implementing network protocols. IEEE Trans. Software Eng., 17(1):64-76, Jan. 1991.

[8] H. Meling. Adaptive Middleware Support and Autonomous Fault Treatment: Architectural Design, Prototyping and Experimental Evaluation. $\mathrm{PhD}$ thesis, Norwegian University of Science and Technology, Department of Telematics, May 2006.

[9] H. Meling, A. Montresor, B. E. Helvik, and O. Babaoglu. Jgroup/ARM: a distributed object group platform with autonomous replication management. Software Prac. Exper., 38(9):885-923, July 2008.

[10] H. Miranda, A. Pinto, and L. Rodrigues. Appia, a flexible protocol kernel supporting multiple coordinated channels. In Proc. 21st ICDCS, Apr. 2001.

[11] A. Montresor. System Support for Programming Object-Oriented Dependable Applications in Partitionable Systems. PhD thesis, Dept. of Computer Science, University of Bologna, Feb. 2000.

[12] O. Rütti, P. T. Wojciechowski, and A. Schiper. Service Interface: A New Abstraction for Implementing and Composing Protocols. In Proc. 21st ACM Symp. on Applied Computing, Dijon, France, Apr. 2006.

[13] P. Urbán, X. Défago, and A. Schiper. Neko: A single environment to simulate and prototype distributed algorithms. Journal of Information Science and Engineering, 18(6):981-997, Nov. 2002.

[14] R. van Renesse. Masking the Overhead of Layering. In Proc. 1996 ACM SIGCOMM, Aug. 1996.

[15] R. van Renesse, K. P. Birman, and S. Maffeis. Horus: A Flexible Group Communication System. Communications of the ACM, 39(4):76-83, Apr. 1996. 